\title{
Quantitative Analysis of Locomotive Behavior of Human Sperm Head and Tail
}

\author{
Jun Liu, Student Member, IEEE, Clement Leung, Member, IEEE, Zhe Lu, Member, IEEE, \\ and Yu Sun*, Senior Member, IEEE
}

\begin{abstract}
Sperm selection plays a significant role in in vitro fertilization (IVF). Approaches for assessing sperm quality include noninvasive techniques based on sperm morphology and motility as well as invasive techniques for checking DNA integrity. In 2006, a new device using hyaluronic acid (HA)-coated dish for sperm selection was cleared by the Food and Drug Administration (FDA) and entered IVF clinics. In this technique, only sperms with DNA integrity bind to the HA droplet, after which these bound sperm stop revealing head motion and their tail movement becomes more vigorous. However, selecting a single sperm cell from among HA-bound sperms is ad hoc in IVF clinics. Different from existing sperm tracking algorithms that are largely limited to tracking sperm head only and are only able to track one sperm at a time, this paper presents a multisperm tracking algorithm that tracks both sperm heads and low-contrast sperm tails. The tracking results confirm a significant correlation between sperm head velocity and tail beating amplitude, demonstrate that sperms bound to $\mathrm{HA}$ generally have a higher velocity (before binding) than those sperms that are not able to bind to HA microdots, and quantitatively reveal that HA-bound sperms' tail beating amplitudes are different among HA-bound sperms.
\end{abstract}

Index Terms-Beating amplitude, hyaluronic acid (HA), multiple sperm tracking, sperm selection, sperm tail.

\section{INTRODUCTION}

$\mathbf{T}$ HE mechanisms of natural sperm selection are not well understood. It is accepted, however, that the sperm selection mechanisms play a great significant role for the inheritance of superior health traits such as disease resistance, offspring survival, and fecundity [1], [2]. In natural human conception, sperm selection occurs as a healthy sperm actively seeks out and fertilizes an egg. However, for couples having infertility issues, assisted reproduction technologies are required to address their reproductive needs. For instance, in intracytoplasmic sperm injection (ICSI), an embryologist selects a single sperm and injects it into an oocyte (i.e., egg cell) to overcome issues such as male infertility [3]. This IVF procedure bypasses the physiologic and

Manuscript received June 4, 2012; revised September 1, 2012 and October 23, 2012; accepted October 28, 2012. Date of publication November 16, 2012; date of current version January 16, 2013. This work was supported in part by the Natural Sciences and Engineering Research Council of Canada and in part by the Ontario Centres of Excellence. Asterisk indicates corresponding author

J. Liu, C. Leung, and Z. Lu are with the Advanced Micro and Nanosystems Laboratory, University of Toronto, Toronto, ON M5S 3G8, Canada (e-mail: ljun@mie.utoronto.ca; clement.leung@utoronto.ca; zhe.lu@utoronto.ca).

*Y. Sun is with the Advanced Micro and Nanosystems Laboratory, University of Toronto, Toronto, ON M5S 3G8, Canada (e-mail: sun@mie.utoronto.ca).

Color versions of one or more of the figures in this paper are available online at http://ieeexplore.iee.org.

Digital Object Identifier 10.1109/TBME.2012.2227319 biologic barriers for sperm selection and demands the operator to select high-quality sperms.

The criteria for sperm assessment provided by the World Health Organization are vitality, morphology, and motility [4]. In IVF, sperm selection is commonly based on sperms' motility and morphology attributes. A widely used method for sperm selection is motile sperm organelle morphology examination [5], [6]. Sperm motility is also a widely accepted criterion for sperm quality assessment. The past few decades have witnessed the development of computer-assisted sperm analysis (CASA) methods for measuring both sperm morphology and motility [7].

Recently, an emerging methodology was introduced for selecting viable sperms with a high level of DNA integrity. In order to noninvasively select a healthy sperm, Huszar's group proposed the use of a hyaluronic acid (HA) assay [8]. HA is a linear polysaccharide in the extracellular matrix of cumulus oophorus around the oocyte and plays an important role in natural human fertilization [9]. A series of studies on HA-based sperm selection confirmed that the HA assay is able to select healthy sperms with no DNA damage [10], [11], and has received FDA approval. In the HA assay, sperms that bind to HA microdots are proven to have a higher level of DNA integrity compared to those unbound sperms. When a sperm binds its head to an HA microdot, the sperm loses its progressive movement and the tail beating motion becomes more vigorous. The sperm tail beating amplitude becomes the only parameter to differentiate the HA bound sperms from each other, calling for techniques to quantify HA-bound sperms' tail beating motion.

Several algorithms have been reported for tracking multiple moving objects. Model-based tracking algorithms incorporate priori information about the objects to develop representations such as skin complexion [12], shape [13], kinematic skeleton [14], silhouettes [15], or layer information [16]. Appearance-based approaches apply recognition algorithms to track objects in eigenspace [17] derived from observations or in kernel space [18]. The correspondence of multiobject tracking becomes complex with the presence of occlusions, misdetections, entries, and exits of objects. Algorithms for solving the problems of nearby confuser and objects occlusion/overlap include deterministic methods [19], single object state estimation (e.g., Kalman filter [20], particle filter [21]), and multipleobject data association and state estimation (e.g., global nearest neighbor method [22] and joint probability data association filter [23]). Due to color uniformity and shape similarity of sperms, multisperm tracking has not been well studied.

Several algorithms have been developed to track sperm trajectories, measure sperm velocities, and evaluate sperm energetics [24]-[26]. Shi et al. reported a single-sperm tracking 
algorithm based on a four-class thresholding method to extract a single sperm in a small region of interest [27]. The method is limited to tracking a single sperm and is incapable of multisperm tracking. Nafisi et al. demonstrated a template matching algorithm for sperm tracking. The algorithm is insensitive to image acquisition conditions [28]. However, this algorithm relies on user input to obtain the sperm's initial position and cannot track multiple sperms. Existing algorithms for sperm tracking are largely limited to sperm head tracking. The small size ( $\leq 1 \mu \mathrm{m}$ in thickness) and low contrast of sperm tails under optical microscopy make sperm tail tracking challenging. In our previous study [29], a maximum intensity region algorithm was developed for sperm tail tracking. The tracking algorithm, without proper filtering, can be susceptible to disturbances, such as overlapping of the target sperm with other sperms or debris and changes in lighting conditions.

In this paper, we report an approach for tracking both sperm head and tail. We use a motion template method to detect and track multiple moving sperms, and integrate a Kalman filter to the maximum intensity region algorithm to locate the sperm tail's position. With the positions of the sperm head and tail detected, the sperm's velocity and tail beating amplitude were measured. Experimental results demonstrate that there is a significant correlation between the sperm velocity and its tail beating amplitude. We also analyzed sperm motility and the tail beating movement on HA coated dishes. We found that sperms with a higher level of motility are more likely to bind to the HA microdots, and the sperm tail beating amplitude significantly increases after a sperm binds to the HA microdots. Quantitative analysis of sperm tail's beating amplitude can provide useful information for sperm selection.

\section{EXPERIMENTAL SETUP AND DESIGN}

\section{A. Experimental Setup}

Human sperm samples were tested under a standard inverted microscope (bright field imaging, Nikon TE2000-S). A $20 \times$ objective with a numerical aperture of 0.45 was used (CFI Plan Fluor ELWD, Nikon). A CMOS camera (601f, Basler; resolution: $640 \times 480$ ) was connected to the microscope to capture images at a frame rate of 30 frame/s.

\section{B. Experimental Design}

We first investigated the correlation between sperm velocity and sperm tail beating amplitude. In this experiment, human sperms were placed in a Petri dish containing a standard medium (SpermCatch, NidaCon International). Mineral oil was used to cover the medium to prevent evaporation. Fig. 1(a) and (b) shows a sperm changed its position between the two image frames. Sperms that had linearly progressive movement for at least $2 \mathrm{~s}$ were taken into consideration. To evaluate the linearity of the sperm motion, sperm's average VCL and straight line velocity were calculated by measuring the sperm's head position in each frame. Additionally, the sperm tail beating amplitude was measured using the sperm tail tracking algorithm.
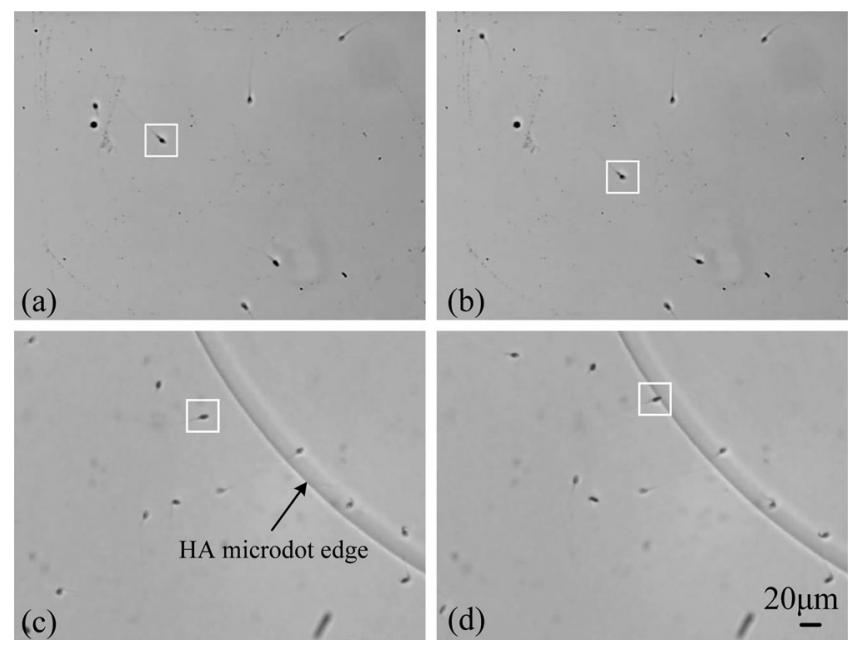

Fig. 1. Experiment design. (a) and (b) Experiment 1: a sperm changed its position between two frames. (c) and (d) Experiment 2: a sperm was moving toward an HA microdot and then bound to the HA microdot.

The second experiment was designed to investigate the difference of sperm velocity and tail beating amplitude between HA bound sperms and those unbound ones. Human sperms were analyzed in a PICSI dish (MidAtlantic diagnostic, Mount Laurel, NJ) with HA microdots coated at the dish bottom. The microdots were first hydrated by placing $10 \mu \mathrm{l}$ droplets of human tubal fluid, and were added with $10 \mu \mathrm{l}$ SpermCatch. Human sperm was then placed on the HA microdots, which were covered with mineral oil to prevent the culture medium from evaporation. The experiment was conducted at room temperature. Some sperms were observed to bind to the microdots after 5 min. Fig. 1(c) shows that a sperm was moving toward an HA microdot, and Fig. 1(d) shows the same sperm bound to the HA microdot. When a sperm bound to the HA microdot, its head motion stopped and its tail beat vigorously. In contrast, sperms that might have DNA defects swam freely on top of the HA microdots without binding. In this experiment, the head velocity of HA bound sperms before binding and the head velocity of unbound sperms were measured and compared. The increase of sperm tail beating amplitude after a sperm binds to an HA microdot was also measured.

\section{SPERM TRACKING}

\section{A. Overview}

In both experiments described in the previous section, the sperm velocity and tail beating amplitude are calculated in every frame of image. The algorithm consists of three steps. The first step tracks the sperm head and uses its position to calculate the sperm curvilinear velocity (VCL), straight line velocity (VSL), and linearity of the sperm's moving path. In the second step, the sperm tail region of interest (STROI) is extracted. STROI extraction is an extrapolation process that calculates the region in which the sperm tail is located by using information from the first step. The STROI is used to capture the tail tip region of the sperm. Once the STROI is found, the maximum intensity region (MIR) algorithm is used to locate a point on the sperm 


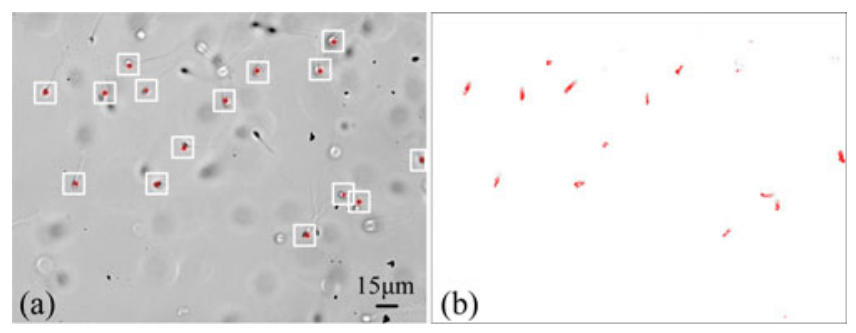

Fig. 2. Tracking multiple sperm heads. (a) Red dots are tracked sperm heads. (b) Corresponding motion history image.

TABLE I

DYNAMIC REPRESENTATION OF SPERM POSITIONS IN THE MULTISPERM TRACKING ALGORITHM

\begin{tabular}{|c|c|c|c|c|c|}
\hline $\begin{array}{l}\text { Position Sperm } \\
\text { Frame }\end{array}$ & 1 & 2 & $\ldots$ & $J$ & $J+1$ \\
\hline$i$ & $P_{i 1}$ & $P_{i 2}$ & $\ldots$ & $P_{i J}$ & \\
\hline$i+1$ & $P_{i+1,1}$ & $P_{i+1,2}$ & $\ldots$ & $P_{i+1, J}$ & \\
\hline$i+2$ & $P_{i+2,1}$ & & $\ldots$ & $P_{i+2, J}$ & \\
\hline$i+3$ & $P_{i+3,1}$ & & $\ldots$ & $P_{i+3, J}$ & $P_{i+3, J+1}$ \\
\hline . & & & $\vdots$ & $\cdot$ & \\
\hline
\end{tabular}

tail within the STROI. Finally, a Kalman filter is used to improve the accuracy of the located point on the sperm tail.

\section{B. Sperm Head Tracking}

There are typically multiple sperms moving randomly within a field of view. In order to detect the sperm heads simultaneously, a multitarget tracking algorithm was developed. For a specific single sperm, a silhouette image of this sperm is obtained by subtracting two consecutive frames. The silhouette image is then binarized by applying a threshold (i.e., 60) to suppress the background noise. When this sperm moves, new silhouettes are captured and overlaid to the old silhouette that fades over time. The time duration of every silhouette is set to be $0.5 \mathrm{~s}$. The sequentially fading silhouettes record the motion history of this sperm. Using this method, a motion history image (MHI) [30] of all the moving objects in the same field of view is obtained, as shown in Fig. 2(b). The position of each moving object is calculated from its central moment in the MHI. Among these moving objects, there are some objects with very little motion caused by those sperms with extremely low motility or by Brownian motions of debris. These objects are excluded by applying morphological transformations (i.e., erosion and dilation) to the MHI. Fig. 2(a) shows the detected moving sperms.

To track multiple sperms, the position history of each sperm is recorded and managed in the multiple sperm tracking algorithm, as summarized in Table I. When $J$ sperms are detected at frame $i, P_{i j}$ represents the position of the $j$ th sperm at frame $i$. When a sperm moves close to the edge of the image and its moving direction is toward the boundary, it is considered swimming out of the field of view. The algorithm then terminates the corresponding data column for this sperm. As shown in Table I, the second column disappears after frame $i+1$, which means that the second sperm has moved out of the field of view. On the other hand, when a sperm newly moves into the field of view, it is added into the data column. For instance, a new sperm moves into the field of view at frame $i+3$. Correspondingly, a new column is added (see the last column in Table I).

To track an individual sperm, the direction vector of the sperm is used as a unique identifier to distinguish it from other sperms with similar color and shape. By denoting the position of the $j$ th sperm at $i+1$ frame as $P_{i+1, j}$, the sperm's direction vector is $D_{i j}=P_{i+1, j}-P_{i, o}$, where $o \in[1, J]$. We found experimentally that the maximum moving distance of fast sperms between two consecutive frames is approximate $3.86 \mu$ m (i.e., 8 pixels). Therefore, if the distance between the $j$ th sperm's position at frame $i+1$ and the oth sperm's position at frame $i$ is less than 8 pixels (i.e., $\left\|D_{i j}\right\|<8$ ), these two sperms are recognized as the same sperm (i.e., $o=j$ ). There may be instances in which two or more sperms at frame $i+1$ have a distance less than 8 pixels to the $j$ th sperm in frame $i$. The average movement direction in previous 30 frames, $\overline{D_{j}}$, is then used as a unique identifier to determine which sperm is the correct $j$ th sperm

$$
\overline{D_{j}}=\frac{1}{30} \sum_{k=1}^{30} D_{i-k, j} .
$$

The candidate sperm $s$ that produces the minimum Euclidean distance value is considered the same $j$ th sperm in the previous frame $i$

$$
s=\min _{m \in[1, M]}\left\|D_{m}-\overline{D_{j}}\right\|
$$

where $M$ is in frame $i+1$ the total number of sperms close to the $j$ th sperm (i.e., $\left\|D_{i j}\right\|<8$ ) and $D_{m}$ is the distance vector between the candidate sperm and the $j$ th sperm.

Assume that the $j$ th sperm enters the field of view at frame $i$ and swims out of the field of view at frame $i+N$. With the sperm position detected in each frame, the travel distance of the $j$ th sperm between two consecutive frames can be determined from its direction vector $D_{i j}$. The VCL, which is the average velocity of the sperm head along its actual curvilinear path, is

$$
\mathrm{VCL}_{j}=\frac{1}{N} \sum_{k=0}^{N-1} D_{i+k, j} .
$$

The VSL, which is the average velocity of the sperm head along the straight line between its first and last detected position, is

$$
\mathrm{VSL}_{j}=\frac{P_{i+N, j}-P_{i j}}{N} .
$$

The linearity (LIN) of the sperm's curvilinear path is

$$
\mathrm{LIN}=\frac{\mathrm{VSL}}{\mathrm{VCL}}
$$

where LIN is the linearity measure $(0 \leq \operatorname{LIN} \leq 1)$. A higher LIN value means that the sperm's moving path is more linear. Healthy energetic sperms with progressive/linear movement are desired (versus those traveling in circles for instance) in sperm selection. In our experiments, only those sperms having a LIN value greater than 0.9 were considered for further analysis. 


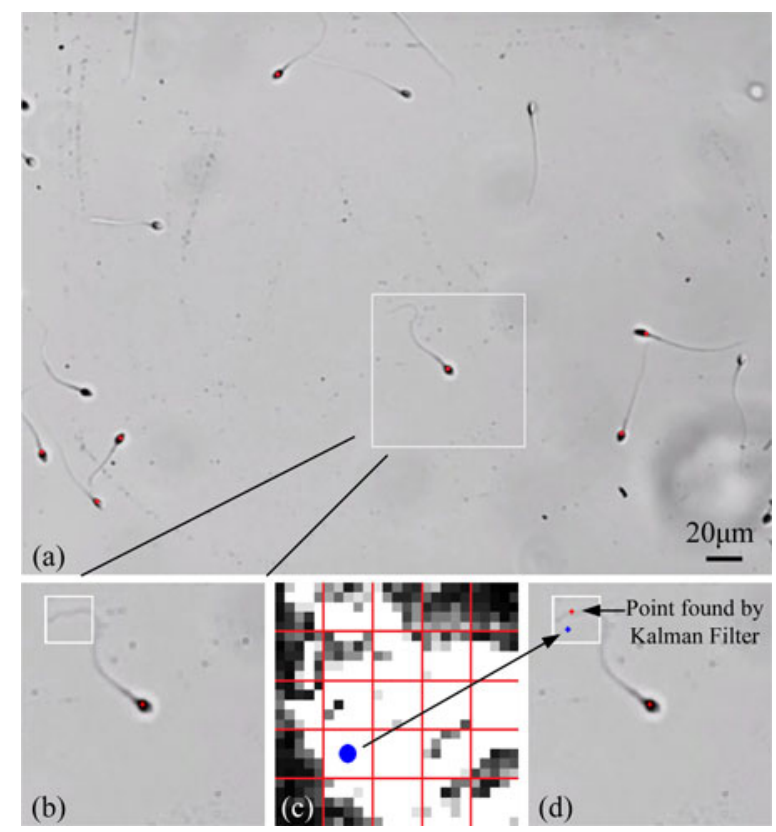

Fig. 3. Sperm tail tracking. (a) Sperm head position is found. (b) STROI is determined. (c) $5 \times 5$ windows are scanned to locate the section with the highest intensity sum in the flicker image. The center point (blue dot in figure) of the section is considered the tail location. (d) Based on the blue dot position found in the flicker image, Kalman filter is applied to improve the accuracy of located sperm tail position.

\section{Sperm Tail Tracking}

After the sperm head position is detected, the sperm tail tracking algorithm extracts an STROI. As shown in Fig. 3(b), the STROI is determined using the sperm head position and the average direction vector of its movement. The average direction vector $\overline{D_{j}}$ is used instead of the direction vector $D_{i j}$ because the sperm may exhibit abrupt changes in movement direction between two consecutive frames. By averaging the direction vectors of the sperm across a number of frames (e.g., 30 frames), the effect of abrupt changes in the sperm moving direction between frames is mitigated and the extraction of STROI becomes more robust.

The STROI's center position in the $i$ frame $T_{i j}$ is determined by subtracting a scaled value of the direction vector from the sperm head's centroid

$$
T_{i j}=P_{i j}-a \cdot \frac{\overline{D_{j}}}{\left\|\overline{D_{j}}\right\|}
$$

where $a$ is a scalar value determined by the human sperm length. Under the $20 \times$ magnification, the average length of human sperms is approximately 90 pixels (i.e., $a=90$ ). After the center position is found, a $25 \times 25$ region of interest is taken as the STROI. The size of $25 \times 25$ provides a sufficient tail search area that takes into consideration a range of sperm tail length variations and sperm tail beating amplitudes.

After finding the STROI, the algorithm verifies that a tail is present in the STROI. The fundamental feature of flicker is extracted by taking the absolute difference between several consecutive inverted grayscale image frames. A higher number of frames to form the flicker image results in more enhancement of the image contrast. On the other hand, too many consecutive frames would add too much sperm tail's history information that can influence the sperm tail tracking accuracy. Experimentally, we determined that six consecutive frames were appropriate to use for forming a flicker image

$$
f(i)=\sum_{k=0}^{5}|I(i-k)-I(i-k-1)|
$$

where $f(i)$ is the flicker image extracted at frame $i$, and $I$ represents the grayscale images containing the sperm of interest in frame $i$ to frame $i-5$. Each pixel in the flicker image is squared to enhance the pixel values of areas in which the tail is present. The sum of pixel value in the STROI of the $f(i)$ image is used as a measure to determine the presence of a sperm tail. If the pixel sum is above a specified threshold value, a tail is considered present. The threshold value was found experimentally by comparing the pixel sum values of STROI images in which a tail exists against cases where no tail exists. An example flicker image is shown in Fig. 3(c). If the pixel sum is below a threshold value, no tail is found inside the STROI. This situation can occur when the sperm of interest moves out of focus, resulting in the disappearance of the sperm tail.

Once the sperm tail is determined to exist within the STROI, the MIR algorithm uses the flicker image to locate a point on the sperm tail. By extracting the flicker feature of the sperm tail, as shown in Fig. 3(c), the position of the sperm tail can be detected. This approach overcomes the challenges that arise from the lowcontrast image of the sperm tail in a single frame. The algorithm first finds the location of maximum intensity within the $25 \times 25$ STROI of the flicker image. This is accomplished by evaluating the sum of the intensity values inside a $5 \times 5$ window at a spatial sampling interval of 5 pixels in both the $x$ and $y$ coordinates of the STROI flicker image. The center position of the $5 \times 5$ window with the highest intensity is considered the tail location (i.e., a point on the sperm tail).

The located point on the sperm tail is often inaccurate because the flicker image contains noises caused by some dark debris or by other sperms entering the STROI. Therefore, a Kalman filter is applied to correct the measured point on the sperm tail. In order to model the sperm tail motion, the sperm tail's location and velocity in the image coordinate are chosen as state variables (i.e., $X=\left[\begin{array}{llll}x & y & \Delta & \Delta\end{array}\right]^{T}$ ). The model of the sperm tail motion is

$$
X_{k}=A X_{k-1}+w_{k}
$$

where

$$
A=\left[\begin{array}{llll}
1 & 0 & 1 & 0 \\
0 & 1 & 0 & 1 \\
0 & 0 & 1 & 0 \\
0 & 0 & 0 & 1
\end{array}\right]
$$

and $w_{k}$ is noises affecting the actual state of the sperm and is assumed to have a Gaussian distribution, $\left(N\left(0, Q_{k}\right)\right)$.

The sperm tail's position is calculated according to

$$
Z_{k}=H X_{k}+v_{k}
$$


TABLE II

Performance Comparison BetweEn MHI-Based MultisPerm Tracking AND GNN-BASED MULTISPERM TRACKING

\begin{tabular}{c|ccccc}
\hline Algorithms & ATE & FP & FN & FIT & FIO \\
\hline MHI & 0.85 & 3 & 15 & 33 & 28 \\
\hline Shape \& GNN & 1.23 & 21 & 7 & 54 & 48 \\
\hline
\end{tabular}

where

$$
H=\left[\begin{array}{llll}
1 & 0 & 0 & 0 \\
0 & 1 & 0 & 0
\end{array}\right]
$$

and $v_{k}$ is the measurement noise, which is also assumed to have a Gaussian distribution $\left(N\left(0, R_{k}\right)\right) . R_{k}$ is chosen based on the estimate of how accurately the sperm tail's positions are detected using the MIR algorithm.

Based on the dynamic model, a priori estimate of the state is computed $\left(X_{k \mid k-1}=A X_{k-1 \mid k-1}+w_{k}\right)$. The error covariance is denoted as $P_{k \mid k-1}$. The priori estimate for this covariance at time $k$ is then determined by

$$
P_{k \mid k-1}=A P_{k-1 \mid k-1} A^{T}+Q_{k} .
$$

With the priori estimate of the state $X_{k \mid k-1}$ and the measurement $Z_{k}$ (i.e., detected results using the MIR algorithm), the real state of sperm tail is optimized by

$$
X_{k \mid k}=X_{k \mid k-1}+K\left(Z_{k}-H X_{k \mid k-1}\right)
$$

where $K$ is the Kalman gain and is given by

$$
K=\frac{P_{k \mid k-1} H^{T}}{H P_{k \mid k-1} H^{T}+R_{k}} .
$$

After the optimized sperm tail's position is found, the sperm tail beating amplitude inside the STROI is computed. The relative position inside the STROI in frame $i$ is denoted by $P T_{i}$. The sperm tail beating amplitude $A$ is

$$
A=\frac{1}{N} \sum_{i=1}^{N}\left\|P T_{i}-\overline{P T}\right\|
$$

where $\overline{P T}$ is the sperm tail's average position inside the STROI and $N$ is the number of frames until when the sperm tail is successfully detected.

\section{EXPERIMENTAL RESULTS AND DisCUSSION}

The multisperm head tracking algorithm based on MHI was evaluated and compared with the multiple-object tracking algorithm which uses sperm head shape and the global nearest neighbor (GNN) data association method [22]. Both algorithms were applied to the same video clips (476 s in duration) in which more than 200 sperms were present. The evaluation criteria are average tracking errors (ATE), false positive (FP) rate, false negative (FN) rate, false identified trackers (FIT), and false identified objects (FIO), as defined in [31].

As summarized in Table II, the average tracking error of MHI-based algorithm (0.85 pixel) is less than that of shapebased detection algorithm (1.23 pixel). Shape-based multisperm tracking algorithm also has a higher FP rate than the MHI-based

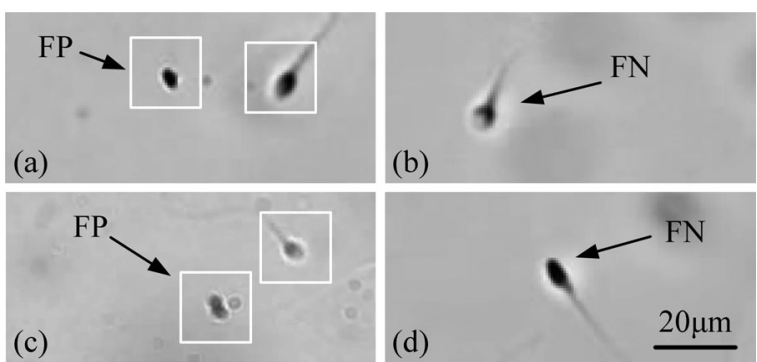

Fig. 4. Failure cases in sperm head tracking. (a) False positive detection of sperm head in shape-based tracking is caused by the stationary object/particle with a shape similar to sperm head. (b) False negative case: shape-based detection algorithm fails to detect the sperm head that has an abnormal head shape. (c) False positive detection of sperm head in MHI-based detection is caused by moving debris. (d) False negative case: MHI-based detection algorithm fails to detect stationary/slow-moving sperms.

tracking algorithm. The FP cases in shape-based tracking were mainly caused by stationary foreign objects (contaminant particles) with a similar shape as the sperm head [see Fig. 4(a)]. Since these objects were largely stationary, they had little effect on MHI-based tracking. The FP cases in MHI-based tracking were caused by cell debris moving with the fluidic flow when sperms were swimming nearby [see Fig. 4(c)]. The experimental results demonstrate that the shape-based tracking algorithm is more susceptible to background noise and is not always effective in filtering debris/particles that have similar shapes as the sperm head. On the other hand, the MHI-based multisperm tracking approach is more robust to background noise and disturbances from foreign stationary particles. The MHI-based tracking algorithm also outperforms the algorithm using GNN data association in terms of FIT and FIO. The GNN data association method only considers sperm head's position in the last frame. Thus, it is not effective in dealing with abrupt movement changes between two consecutive frames. In the MHI-based algorithm, the effect of abrupt changes in sperm movement between frames is mitigated by averaging sperm's movement direction vector across a number of frames.

It needs to be noted, however, that the MHI-based sperm head tracking algorithm has a higher value of FN than the shape-based multisperm tracking algorithm. This is because the MHI-based algorithm cannot detect stationary sperms. In IVF, only motile sperms are of interest for analysis. Therefore, this drawback of MHI-based sperm tracking algorithm does not constrain its applicability in sperm selection. The FN cases in shaped-based and MHI-based algorithms are shown in Fig. 4(b) and (d), respectively.

The sperm tail tracking algorithm was evaluated by measuring the Euclidean distance error between the detected tail point and the actual sperm tail position. The actual position was carefully identified/input by a user for each frame via computer mouse clicking. Table III summarizes the average Euclidean distance error for five additional videos. The overall average Euclidean distance error for the MIR algorithm integrated with Kalman filtering is 1.43 pixels $(0.69 \mu \mathrm{m})$, while the error for the MIR algorithm without Kalman filtering is 1.95 pixels $(0.95 \mu \mathrm{m})$. This result indicates that Kalman filtering is effective in improving 
TABLE III

AVERAGE ERROR OF SPERM TAIL TRACKING

\begin{tabular}{c|ccccc|c}
\hline Video & $\mathrm{V} 1$ & $\mathrm{~V} 2$ & $\mathrm{~V} 3$ & $\mathrm{~V} 4$ & $\mathrm{~V} 5$ & Average \\
\hline Duration (sec) & 3.83 & 6.67 & 3.83 & 4.60 & 3.87 & 4.56 \\
\hline MIR (pixels) & 1.16 & 2.73 & 1.83 & 2.02 & 1.98 & 1.95 \\
\hline With Kalman (pixels) & 0.97 & 2.53 & 1.05 & 1.39 & 1.25 & 1.43 \\
\hline
\end{tabular}

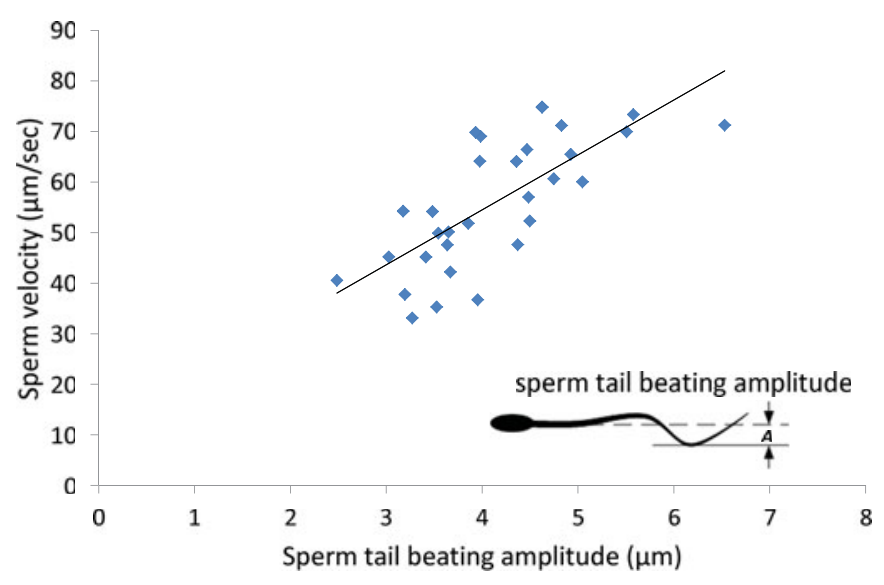

Fig. 5. Correlation between sperm velocity and tail beating amplitude. Pearson's correlation coefficient $r=0.7225$.

the sperm tail tracking accuracies of the MIR algorithm. When the MIR algorithm failed to track the sperm tail due to the overlap with other sperms or occlusion by debris, Kalman filtering was able to estimate the sperm tail's position based on the motion model.

To investigate the correlation between the sperm head velocity and the tail beating amplitude, we chose 30 sperms showing good progressive movements (LIN $>0.9$ ). The tracking algorithms described in Sections III(B) and $\operatorname{III}(\mathrm{C})$ were used to measure their head velocity and the tail beating amplitude. We processed the measured data using a linear regression model. As shown in Fig. 5, there is a statistically significant correlation between the head velocity and sperm tail beating amplitude. Bivariate association between sperm velocity and sperm tail beating amplitude was evaluated by Pearson's correlation coefficient. Tracking data captured on the 30 sperms show that Pearson's correlation coefficient was 0.7225 and the $p$-value was lower than 0.0001 . This result quantitatively demonstrates that the sperm's head velocity is proportional to its tail beating amplitude.

In the HA binding experiment, the heads of the HA bound sperms became stationary, and their tail movements became more vigorous. The process of sperm binding to the HA microdots was recorded at 30 frames/s. Fig. 6(a) and (b) show sperms binding to the HA microdot at 5 and $30 \mathrm{~min}$, respectively. If a moving sperm was detected to stop in the region of HA microdots, this sperm was considered successfully binding to HA. In contrast, if a moving sperm passed the HA microdot and disappeared out of the image boundary, it was considered an HA-unbound sperm. During the process of sperm binding to the HA microdots, the head velocity of the HA-bound sperms

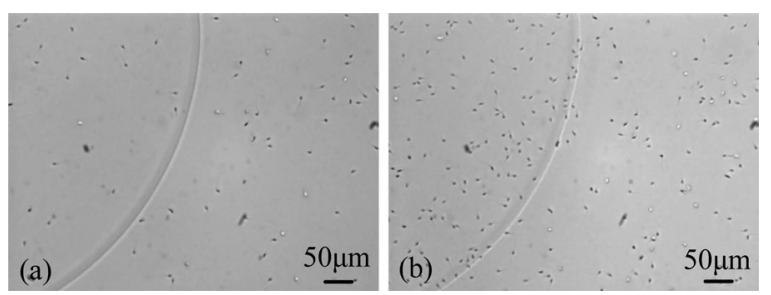

Fig. 6. Sperms on a PICSI dish with HA microdots. (a) Sperms binding after 5 min. (b) Sperms binding after $30 \mathrm{~min}$.

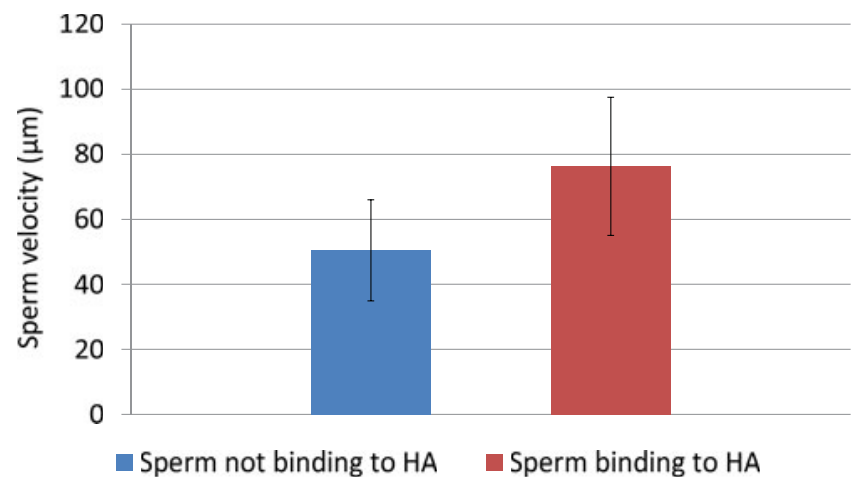

Fig. 7. Velocity comparison between the HA-bound sperms $\left(n_{1}=30\right)$ and unbound sperms $\left(n_{2}=30\right)$.

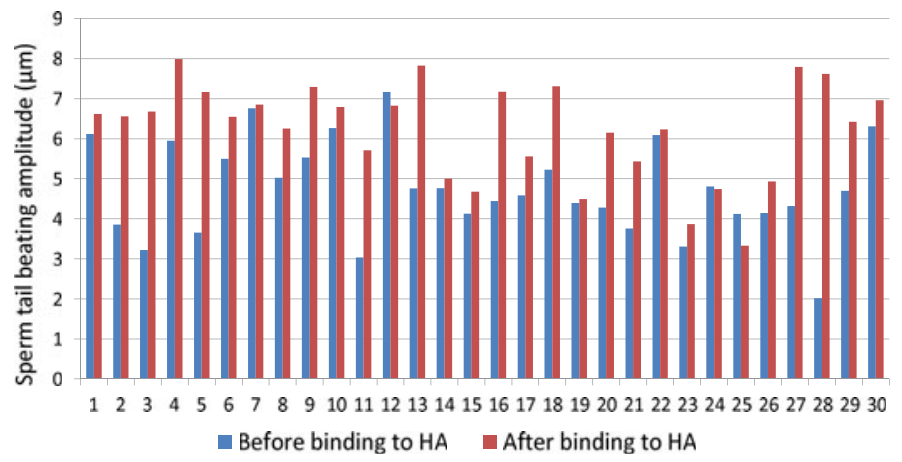

Fig. 8. Tail beating amplitude of the same sperms before and after binding to HA microdots.

before binding was measured. The HA-bound sperms were compared to those unbound ones in terms of their head speed. As in the first experiment, only those sperms that exhibited linearly progressive movement were analyzed. The result, as shown in Fig. 7, demonstrates that the HA-bound sperms tended to have a higher head velocity than those unbound sperms. The HAbound sperms had an average head velocity of $76.28 \mu \mathrm{m} / \mathrm{s}$ with a standard deviation of $21.25 \mu \mathrm{m} / \mathrm{s}$, while the average head velocity of those unbound sperms was $50.45 \mu \mathrm{m} / \mathrm{s}$ with a standard deviation of $15.52 \mu \mathrm{m} / \mathrm{s}$.

We also observed in the HA binding experiment that after the sperms bound to the HA microdots, their tails beat more vigorously. The tail beating amplitude was measured on the same sperms before and after they bound to the HA microdots. Fig. 8 shows sperm tail beating amplitude before and after binding to the HA microdots. The average amplitude produced by these 30 HA-bound sperms was $5.31 \mu \mathrm{m}$ (before binding) and $6.93 \mu \mathrm{m}$ (after binding). These results, for the first time, quantitatively 
reveal an increase in sperm tail beating amplitude before and after a sperm binds to an HA microdot. The results also quantify differences in sperm tail beating amplitude across HA-bound sperms. The measurement of sperm tail beating amplitude can possibly be used as an additional criterion for sperm selection among HA-bound sperms.

\section{CONCLUSION}

This paper presented visual tracking algorithms for tracking both the head and tail of motile human sperms. The sperm head tracking algorithm is capable of tracking multiple moving sperms with a high success rate. Based on the sperm head's position and its motion direction vector, the STROI is located. In this region of interest, the MIR algorithm together with Kalman filtering determines the sperm tail position. The sperm head and tail tracking algorithms enabled a number of new findings. A significant correlation between sperm head velocity and tail beating amplitude was found, suggesting that stronger tail propelling produces a higher velocity. The results also reveal that sperms bound to HA generally have a higher velocity (before binding) than those sperms that are not able to bind to HA microdots. This discovery "unifies" the conventional sperm assessment criterion based on sperm velocity/motility and the most recent HA assay technique. Among the sperms bound to HA microdots, their tails produce different beating amplitudes. Measuring such amplitude differences quantitatively can possibly be used as a new, useful sperm selection criterion among HA bound sperms.

\section{ACKNOWLEDGMENT}

The authors would like to thank the Toronto Centre for Advanced Reproductive Technology for sperm sample preparation.

\section{REFERENCES}

[1] W. Holt and K. J. W. Van Look, "Concepts in sperm heterogeneity, sperm selection and sperm competition as biological foundations for laboratory tests of semen quality," Reproduction, vol. 127, no. 5, pp. 527-535, 2004.

[2] C. H. Ramlau-Hansen, A. M. Thulstrup, J. Olsen, and J. P. Bonde, "Parental subfecundity and risk of decreased semen quality in the male offspring: A follow-up study," Amer. J. Epidemiol., vol. 167, no. 12, pp. 1458-1464, 2008.

[3] G. Palermo, H. Joris, P. Devroey, and A. C. Van Steirteghem, "Pregnancies after intracytoplasmic injection of single spermatozoon into an oocyte," Lancet, vol. 340, no. 8810, pp. 17-18, 1992.

[4] T. G. Cooper, E. Noonan, S. von Eckardstein, J. Auger, H. W. G. Baker, H. M. Behre, T. B. Haugen, T. Kruger, C. Wang, M. T. Mbizvo, and K. M. Vogelsong, "World health organization reference values for human semen characteristics," Human Reprod. Update, vol. 16, no. 3, pp. 231$245,2009$.

[5] B. Bartoov, F. Eltes, M. Pansky, H. Lederman, E. Caspi, and Y. Soffer, "Estimating fertility potential via semen analysis data," Human Reprod., vol. 8, no. 1, pp. 65-70, 1993.

[6] A. Berkovitz, F. Eltes, S. Yaari, N. Katz, I. Barr, A. Fishman, and B. Bartoov, "The morphological normalcy of the sperm nucleus and pregnancy rate of intracytoplasmic injection with morphologically selected sperm," Human Reprod., vol. 20, no. 1, pp. 185-190, 2005.

[7] L. Larsen, T. Scheike, T. K. Jensen, J. P. Bonde, E. Ernst, N. H. Hjollund, Y. Zhou, N. E. Skakkebaek, and A. Giwercman, "Computer-assisted semen analysis parameters as predictors for fertility of men from the general population," Human Reprod., vol. 15, no. 7, pp. 1562-1567, 2000.

[8] G. Huszar, CC. Ozenci, S. Cayli, Z. Zavaczki, E. Hansch, and L. Vigue, "Hyaluronic acid binding by human sperm indicates cellular maturity, viability, and unreacted acrosomal status," Fertil. Steril., vol. 79, no. 3, pp. 1616-1624, 2003.

[9] C. G. Petersen, F. C. Massaro, A. L. Mauri, J. BA. Oliveira, R. LR. Baruffi, and J. G Franco, "Efficacy of hyaluronic acid binding assay in selecting motile spermatozoa with normal morphology at high magnification," Reprod. Biol. Endocrinol., vol. 8, no. 1, pp. 149-155, 2010.

[10] A, Jakab, D. Sakkas, E. Delpiano, S. Cayli, E. Kovanci, D. Ward, A. Revelli, and G. Huszar, "Intracytoplasmic sperm injection: A novel selection method for sperm with normal frequency of chromosomal aneuploidies," Fertil. Steril., vol. 84, no. 6, pp. 1665-1673, 2005.

[11] A. Yagci, W. Murk, J. Stronk, and G. Huszar, "Spermatozoa bound to solid state hyaluronic acid show chromatin structure with high DNA chain integrity: An acridine orange fluorescence study," J. Androl., vol. 31, no. 6, pp. 566-572, 2010.

[12] C. R. Wren, A. Azarbayejani, T. Darrell, and A. Pentland, "Pfinder: Realtime tracking of the human body," IEEE Trans. Pattern Anal. Mach. Intell., vol. 19, no. 7, pp. 780-785, Jul. 1997.

[13] J. Kang, I. Cohen, and G. Medioni, "Object reacquisition using geometric invariant appearance model," in Proc. Int. Conf. Pattern Recong., 2004, pp. $759-762$.

[14] C. Sminchisescu and B. Triggs, "Kinematic jump processes for monocular 3-D human tracking," in Proc. IEEE Conf. Comp. Vis. Pattern Recog., Madison, WI, Jun. 2003, vol. I, pp. 69-76.

[15] G. Cheung, S. Baker, and T. Kanade, "Shape-from-silhouette of articulated objects and its use for human body kinematics estimation and motion capture," in Proc. IEEE Conf. Comp. Vis. Pattern Recog., Madison, WI, Jun. 2003, vol. I, pp. 77-84.

[16] H. Tao, H. S. Sawhney, and R. Kumar, "Object tracking with Bayesian estimation of dynamic layer representations," IEEE Trans. Pattern Anal. Mach. Intell., vol. 24, no. 1, pp. 75-89, Jan. 2002.

[17] M. J. Black and A. D. Jepson, "Eigentracking: Robust matching and tracking of articulated objects using a view-based representation," Int. J. Comput. Vis., vol. 26, no. 1, pp. 63-84, 1998.

[18] D. Comaniciu, V. Ramesh, and P. Meer, "Kernel-based object tracking," IEEE Trans. Pattern Anal. Mach. Intell., vol. 25, no. 5, pp. 564-575, May 2003.

[19] C. Veenman, M. Reinders, and E. Backer, "Resolving motion correspondence for densely moving points," IEEE Trans. Pattern Anal. Mach. Intell., vol. 23, no. 1, pp. 54-72, Jan. 2001.

[20] D. Beymer and K. Konolige, "Tracking people from a mobile platform," Springer Tracts Adv. Robot., vol. 5, pp. 234-244, 2003

[21] M. Isard and A. Blake, "Condensation-Conditional density propagation for visual tracking," Int. J. Comput. Vis., vol. 29, no. 1, pp. 5-28, 1998.

[22] P. Konstantinova, A. Udvarev, and T. Semerdjiev, "A study of a target tracking algorithm using global nearest neighbor approach," in Proc. Int. Conf. Comp. Sys. Tech., New York, 2003, pp. 290-295.

[23] H. A. P. Blom and E. A. Bloem, "Probabilistic data association avoiding track coalescence," IEEE Trans. Autom. Contr, vol. 45, no. 2, pp. 247-259, Feb. 2000.

[24] L. Shi, J. Nascimento, C. Chandsawangbhuwana, E. Botvinick, and M. Berns, "An automatic system to study sperm motility and energetics," Biomed. Microdevices, vol. 10, no. 4, pp. 573-583, 2008.

[25] R. P. Amann and D. F. Katz, "Reflections on CASA after 25 years," $J$. Androl., vol. 25, no. 3, pp. 317-325, 2004.

[26] V. R. Nafisi, M. H. Moradi, and M. H. Nasr-Esfahani, "Sperm identification using elliptic model and tail detection," in Proc. 4th World Enformatika Conf., Istanbul, Turkey, 2005, pp. 205-207.

[27] L.Z. Shi, J. M. Nascimento, M. W. Berns, and E. L. Botvinick, "Computer-based tracking of single sperm," J. Biomed. Opt., vol. 11, no. 5, pp. 054009-054010, 2006.

[28] V. R. Nafisi, M. H. Moradi, and M. H. Nasr-Esfahani, "A template matching algorithm for sperm tracking and classification," Physiol. Meas., vol. 26 , no. 5, pp. 639-651, 2005.

[29] C. Leung, Z. Lu, N. Esfandiari, R. F. Casper, and Y. Sun, "Automated sperm immobilization for intracytoplasmic sperm injection," IEEE Trans. Biomed. Eng., vol. 58, no. 4, pp. 935-942, Apr. 2011.

[30] M. A. Ahad, J. K. Tan, H. Kim, and S. Ishikawa, "Motion history image: Its variants and applications," Mach. Vis. Appl., vol. 23, no. 2, pp. 255-281, 2010

[31] K. Smith, D. Gatica-Perez, J. Odobez, and S. Ba, "Evaluating multi-object tracking," in Proc. IEEE Int. Conf. Comput. Vis. Pattern Recog., Jun. 2005, pp. 36-43.

Authors' photographs and biographies not available at the time of publication. 